\section{Radionuclide Imaging of Infection and Inflammation: A Pictorial Case-Based Atlas}

\author{
E. Lazzeri, A. Signore, P.A. Erba, et al., Eds.
}

New York, NY: Springer, 2013, 347 pages, \$229

This comprehensive pictorial case-based atlas of radionuclide imaging in infection and inflammation comprises a diverse group of excellent cases, many using hybrid SPECT/CT and PET/CT imaging. The cases cover a wide spectrum of infection and inflammation in multiple organ systems from brain to feet, including bone, soft tissues, lung, liver, spleen, heart and major vessels, fever of unknown origin, prosthetic joint and implanted devices, vascular grafts, catheter infections, and infections related to hardware in bone. Infections and inflammation associated with chronic inflammation in diseases such as rheumatoid arthritis, sarcoidosis, Sjögren's disease, vasculitis, and atherosclerosis are also included. The imaging is with commercially and routinely available radiopharmaceuticals such as ${ }^{67} \mathrm{Ga}$-citrate or the newer ${ }^{18} \mathrm{~F}-\mathrm{FDG}$ in PET imaging, and ${ }^{111} \mathrm{In}$-oxine or ${ }^{99 \mathrm{~m}} \mathrm{Tc}-$ hexamethylpropyleneamine oxime (Ceretec; GE Healthcare)-labeled white blood cells, 3-phase bone and sulfur colloid bone marrow scanning, and monoclonal antibodies.

The book is divided into 13 chapters covering a wide spectrum of infection and inflammation imaging in the entire body. It is the end result of collaborative hard work by many medical professionals from medical centers throughout Italy, through their involvement in the study group on inflammation and infection of the Italian Association of Nuclear Medicine. Some cases in this atlas are not routinely or frequently seen in a general nuclear medicine practice and thus can serve as a good go-to reference.

The first chapter is an adequate overview of the radiopharmaceuticals used in infection and inflammation imaging; describes the normal biodistributions, variants, and pitfalls seen during imaging with these different radiopharmaceuticals and agents; and is supplemented by good-quality images. There are extensive references within this chapter for the myriad pitfalls or normal variant findings, which can be further researched if needed.

The second through 13th chapters follow the same format of a brief pertinent introduction or clinical overview of imaging in the specific disease category followed by $2-9$ relevant clinical cases. Many cases use hybrid imaging with mostly ${ }^{99 \mathrm{~m}} \mathrm{Tc}$-hexamethylpropyleneamine oxime-labeled white blood cells and SPECT/CT imaging or ${ }^{18} \mathrm{~F}-$ FDG PET/CT imaging. The image quality throughout the book is good overall, but even more impressive is the wide array of cases.

The section on abdominal infection and inflammation imaging shows many cases of inflammatory bowel disease such as Crohn disease and ulcerative colitis, and the value of the sitting image for evaluating rectal uptake in ulcerative colitis and proctitis is also emphasized. Regarding the ongoing clinical dilemma of differentiating Charcot neuropathy from osteomyelitis in diabetic foot imaging, the superiority and benefit of SPECT/CT imaging is shown in these complicated cases. ${ }^{99 \mathrm{~m}} \mathrm{Tc}$-labeled white blood cell and sulfur colloid marrow imaging in one case shows concordant

COPYRIGHT (C 2013 by the Society of Nuclear Medicine and Molecular Imaging, Inc. uptake due to bone marrow activity, as opposed to the discordant uptake seen in osteomyelitis using these 2 imaging studies. Inclusion of ${ }^{99 \mathrm{~m}} \mathrm{Tc}$-labeled antigranulocyte antibody fragment scanning ( ${ }^{99 m}$ Tc-sulesomab [LeukoScan; Immunomedics, Inc.]) in osteomyelitis and ${ }^{99 \mathrm{~m}} \mathrm{Tc}$-besilesomab (Scintimun; Bayer [labeled monoclonal antibody antigranulocytes]) is useful for those who do not routinely use these agents. The few cases of ${ }^{67} \mathrm{Ga}$ imaging and the chapters on joint infection, brain and head and neck infection, infective endocarditis, and cardiovascular implantable devices show improved anatomic localization of the functional abnormality with SPECT/CT or PET/CT, compared with planar or SPECT images.

The value of 24-h delayed imaging showing a progressive increase in activity in osteomyelitis compared with inflammation is illustrated with good examples. Many good examples of vasculitis are also shown.

The chapter on nonorthopedic or cardiovascular implanted devices has good examples of carotid and skull patch infection, peritoneal dialysis catheter infection, and peritoneal drain infection. The source of infection in fever of unknown origin is identified as liver abscess, bone, soft-tissue or muscle infection, Crohn disease, aortitis, or enterohepatic fistulas.

The cover of the book is appealing, and the glossy pages help to show off the images. Suggested future improvements are the inclusion of more teaching points after the cases so as to reinforce the findings seen in the particular case. Arrows are used in some images, but including arrows in all images will make it easier for newer and younger physicians to more easily see and understand the abnormal finding in the case under discussion.

Overall, this book contains an extensive and impressive collection of infection and inflammation cases in a variety of disease conditions supplemented with good-quality SPECT/CT and PET/CT images. This book is relevant and timely because of the many complicated infectious processes in the many organ systems and because of the increasing use of implanted medical devices, hardware, prostheses, and grafts, complicated by immunodeficiency states, resistant microbial pathogens, and natural aging. The book reinforces that nuclear medicine is moving away from planar or SPECT imaging to hybrid imaging, with its better anatomic localization of the functional abnormality and thus greater helpfulness in patient care. It is apparent that a lot of thought, dedication, and hard work have gone into making this comprehensive and useful atlas on infection and inflammation imaging.

I highly recommend that this atlas be added to any radiology or nuclear medicine departmental library as a good reference manual for scan abnormalities seen on infection and inflammation imaging and covering a wide spectrum of disease processes. This book will help the younger nuclear physician or those in training to learn from and expand their knowledge base and can also serve as a refresher manual for older physicians who may be unfamiliar with some of the abnormalities showcased in this atlas.

\author{
Usha A. Joseph \\ University of Texas Medical School at Houston \\ 6431 Fannin St., MSB 2 \\ Houston, TX 77030 \\ E-mail: usha.a.joseph@uth.tmc.edu
}

Published online Aug. 5, 2013.

DOI: 10.2967/jnumed.113.128710 